\title{
Vasoinhibins prevent retinal vasopermeability associated with diabetic retinopathy in rats via protein phosphatase $2 \mathrm{~A}$-dependent eNOS inactivation
}

\author{
Celina García, ${ }^{1}$ Jorge Aranda, ${ }^{1}$ Edith Arnold, ${ }^{1}$ Stéphanie Thébault, ${ }^{1}$ \\ Yazmín Macotela, ${ }^{1}$ Fernando López-Casillas, ${ }^{2}$ Valentín Mendoza, ${ }^{2}$ \\ Hugo Quiroz-Mercado, ${ }^{3}$ Hebert Luis Hernández-Montiel, ${ }^{4}$ Sue-Hwa Lin, ${ }^{5}$ \\ Gonzalo Martínez de la Escalera, ${ }^{1}$ and Carmen Clapp ${ }^{1}$ \\ ${ }^{1}$ Instituto de Neurobiología, Universidad Nacional Autónoma de México, Querétaro, Mexico \\ IInstituto de Fisiología Celular, Universidad Nacional Autónoma de México, Mexico City, Mexico. ${ }^{3}$ Asociación para Evitar la Ceguera (APEC), \\ Hospital “Dr. Luís Sánchez Bulnes,” Mexico City, Mexico. "Facultad de Medicina, Universidad Autónoma de Querétaro, \\ Querétaro, Mexico. ${ }^{5}$ University of Texas MD Anderson Cancer Center, Houston, Texas, USA
}

\begin{abstract}
Increased retinal vasopermeability contributes to diabetic retinopathy, the leading cause of blindness in working-age adults. Despite clinical progress, effective therapy remains a major need. Vasoinhibins, a family of peptides derived from the protein hormone prolactin (and inclusive of the $16-\mathrm{kDa}$ fragment of prolactin), antagonize the proangiogenic effects of VEGF, a primary mediator of retinal vasopermeability. Here, we demonstrate what we believe to be a novel function of vasoinhibins as inhibitors of the increased retinal vasopermeability associated with diabetic retinopathy. Vasoinhibins inhibited VEGF-induced vasopermeability in bovine aortic and rat retinal capillary endothelial cells in vitro. In vivo, vasoinhibins blocked retinal vasopermeability in diabetic rats and in response to intravitreous injection of VEGF or of vitreous from patients with diabetic retinopathy. Inhibition by vasoinhibins was similar to that achieved following immunodepletion of VEGF from human diabetic retinopathy vitreous or blockage of NO synthesis, suggesting that vasoinhibins inhibit VEGF-induced NOS activation. We further showed that vasoinhibins activate protein phosphatase 2A (PP2A), leading to eNOS dephosphorylation at Ser1179 and, thereby, eNOS inactivation. Moreover, intravitreous injection of okadaic acid, a PP2A inhibitor, blocked the vasoinhibin effect on endothelial cell permeability and retinal vasopermeability. These results suggest that vasoinhibins have the potential to be developed as new therapeutic agents to control the excessive retinal vasopermeability observed in diabetic retinopathy and other vasoproliferative retinopathies.
\end{abstract}

\section{Introduction}

Diabetic retinopathy is a frequent complication in the eye of individuals with long-term diabetes and remains the leading cause of blindness in working-age adults throughout the world. Nearly $80 \%$ of patients develop diabetic retinopathy after the first 2 decades of diabetes (1). The primary pathogenic factor is believed to be chronic hyperglycemia, which causes apoptosis of pericytes and endothelial cells, thickening of vessel basement membranes, and increased retinal vasopermeability. Elevated retinal vasopermeability results in retinal hemorrhages, swelling, and formation of exudates that impair vision when the macula is affected $(2,3)$. Over time, intraretinal hemorrhages and capillary closure create areas of nonperfusion that lead to localized hypoxia and increased production of proangiogenic factors in the retina. In advanced disease, the neovessels invade and bleed into the vitreous, producing a fibrovascular tissue that can cause retinal detachment

Nonstandard abbreviations used: BAEC, bovine aortic endothelial cell; L-NAME $N^{\omega}$-nitro-L-arginine methyl ester; OA, okadaic acid; PP2A, protein phosphatase $2 \mathrm{~A}$; PRL, prolactin; RRCEC, rat retinal capillary endothelial cell.

Conflict of interest: The authors have declared that no conflict of interest exists. Citation for this article: J. Clin. Invest. 118:2291-2300 (2008). doi:10.1172/JCI34508. and blindness. Laser photocoagulation is often effective to treat diabetic retinopathy but rarely improves visual acuity (4). Because laser photocoagulation damages neural tissue, vision can thereby even become worse (4). Thus, developing new strategies to oppose excessive retinal vasopermeability and angiogenic responses has become a major research focus.

VEGF is a prominent vasopermeability factor in diabetic retinopathy $(5,6)$. Intraocular injection of VEGF induces vasodilation, retinal hemorrhages, and vascular leakage in nondiabetic animals (7-10). Elevated concentrations of VEGF in diabetic retinas are detected in association with excessive retinal vasopermeability (11), and therapies based on the inhibition of VEGF expression and action have shown promising results as an effective and safe option for the treatment of diabetic macular edema (6). Both paracellular and transcellular pathways of vascular solute flux have been proposed for VEGF-induced vasopermeability: VEGF alters endothelial cell tight junctions (12) by redistributing occludin (13), but it can also induce formation of caveolae within endothelial cells (14). The latter action involves activation of eNOS and production of $\mathrm{NO}$, a free radical known to be the main endothelium-derived relaxing factor and a mediator of vasopermeability (14-16). Notably, VEGF does not stimulate vasopermeability in 
A

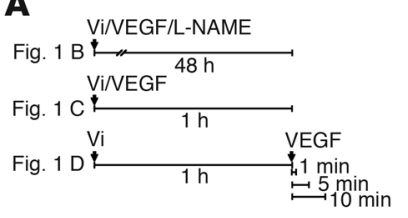

B

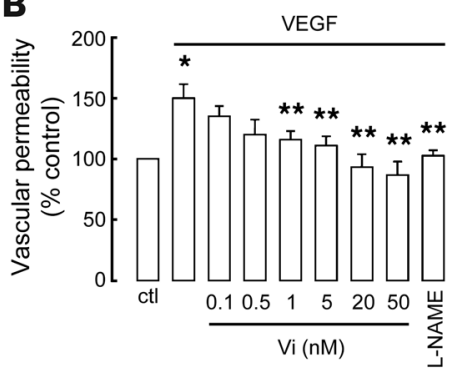

C

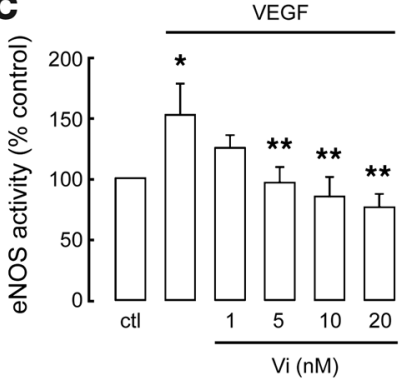

$\mathbf{E}$

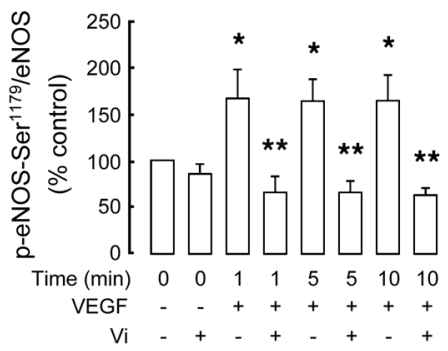

Figure 1

Vasoinhibins (Vi) prevent VEGF-induced vasopermeability, eNOS activity, and eNOS phosphorylation in endothelial cells. (A) Line diagrams depicting the different protocols used in experiments represented in B, C, and D. (B) Vasopermeability was measured by HRP leakage in BAEC monolayers either untreated (ctl) or treated with $5 \mathrm{nM}$ VEGF in the absence or presence of $0.1,0.5,1,5,20$, or $50 \mathrm{nM}$ vasoinhibins for 48 hours. BAECs were incubated with VEGF combined with $1 \mathrm{mM}$ L-NAME. Values are mean \pm SEM of 5 independent experiments. ${ }^{*} P<0.05$ versus ctl; ${ }^{* \star} P<0.05$ versus VEGF. (C) eNOS activity was determined by the $\left[{ }^{3} \mathrm{H}\right] \mathrm{L}$-citrulline assay in BAECs incubated or not with $5 \mathrm{nM}$ VEGF, alone or combined with increasing concentrations of vasoinhibins for 1 hour. Values are mean \pm SEM of 5 independent experiments. ${ }^{*} P<0.05$ versus no treatment; ${ }^{*} P<0.05$ versus VEGF. (D) Western blot analysis of eNOS-Ser1179 phosphorylation in BAECs preincubated or not with $20 \mathrm{nM}$ vasoinhibins for 1 hour and then treated with $5 \mathrm{nM}$ VEGF for 1, 5, or 10 minutes. Total eNOS served as loading control. (E) Quantification of eNOS phosphorylation by densitometry after normalization for total eNOS. Values are mean $\pm \mathrm{SEM}$ of 3 independent experiments. ${ }^{\star} P<0.05$ versus no treatment; ${ }^{*} P<0.05$ versus VEGF.

eNOS ${ }^{-/-}$mice (17), and NOS inhibitors prevent retinal hyperpermeability induced by VEGF in rats (18).

Vasoinhibins are a family of peptides derived by proteolytic cleavage from the hormone prolactin (PRL) (reviewed in ref. 19). These peptides efficiently prevent VEGF-related angiogenesis and promote apoptosis-mediated vascular regression, as observed, for example, in retinopathy of prematurity (20). Vasoinhibins also inhibit bradykinin- and acetylcholine-induced relaxation of coronary vessels via $\mathrm{Ca}^{2+}$-dependent eNOS inactivation (21). Of importance, vasoinhibins are produced in the retina, and the intravitreous injection of siRNA for PRL elicited not only angiogenesis, but also vasodilation (22).

In this study, we determined the effect of vasoinhibins on diabetic retinopathy-associated retinal vasopermeability. We showed that vasoinhibins inhibit VEGF-induced vasopermeability in 2 endothelial cell cultures (bovine aortic endothelial cells [BAECs] and rat retinal capillary endothelial cells [RRCECs]). We further demonstrated that vasoinhibins block vasopermeability induced by intravitreous injection of VEGF or human diabetic vitreous in rat retinas as well as in diabetic rats. Moreover, we identified what we believe to be a novel mechanism by which vasoinhibins prevent the retinal vasopermeability that accompanies diabetic retinopathy.

\section{Results}

Vasoinhibins prevent VEGF-induced endothelial permeability and promote eNOS dephosphorylation at Ser1179. We investigated the effect of VEGF and vasoinhibins on vasopermeability by measuring leakage of HRP across BAEC monolayers. VEGF induced a $50 \%$ increase in permeability, and this increase was blocked in a dose-dependent manner by the coadministration of recombinant vasoinhibins (Figure $1 \mathrm{~B}$ ). Because $\mathrm{NO}$ is one of the main factors mediating the VEGF effect on vasopermeability $(17,18)$, we found that addition of the NOS inhibitor $N^{\omega}$-nitro-L-arginine methyl ester (L-NAME) (23) reduced VEGF-induced BAEC permeability to levels comparable to those of untreated or vasoinhibin-treated cells (Figure 1B). To determine whether vasoinhibins inhibited endothelial cell permeability by blocking NO production, the effect of vasoinhibins on eNOS activity was investigated using the $\left[{ }^{3} \mathrm{H}\right] \mathrm{L}$-citrulline assay in BAECs. Vasoinhibins prevented VEGF-induced activation of eNOS in a dose-dependent manner (Figure 1C). Because eNOS activation occurs through PI3K/Akt-mediated phosphorylation of its Ser1179 (24), we evaluated eNOS phosphorylation in BAECs by Western blot analysis. As shown in Figure 1D, VEGF treatment enhanced eNOS phosphorylation at Ser1179 as compared with no treatment, whereas preincubation with vasoinhibins for 1 hour prior to addition of VEGF blocked the VEGF-induced eNOS phosphorylation. In the presence of vasoinhibins, phosphorylation levels were slightly lower than those of controls (i.e., no treatment or vasoinhibins alone). Figure 1E shows quantification of phosphorylated eNOS after normalization for the amount of total eNOS on the gel. Phosphorylation of eNOS occurred within 1 minute after addition of VEGF, and the level of phosphorylation did not further increase with longer exposure to VEGF (5 and 10 minutes).

Vasoinhibins prevent VEGF stimulation of endothelial permeability via PP2A-induced eNOS dephosphorylation. We further examined 
A

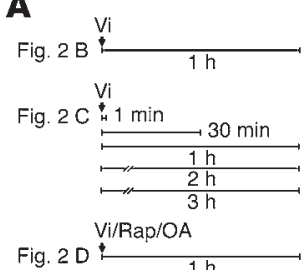

B

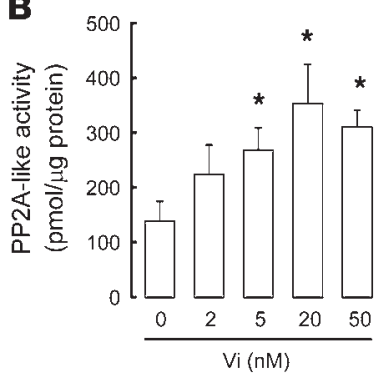

C

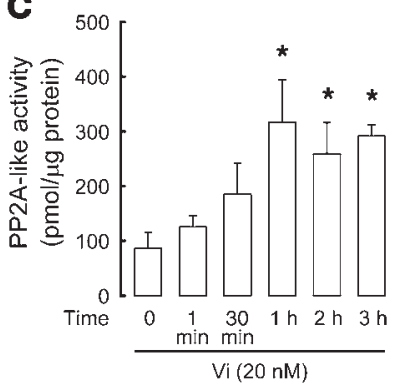

D

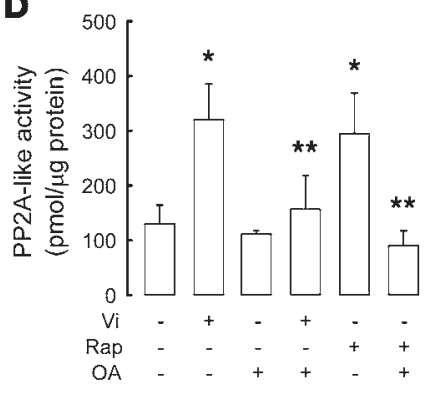

whether vasoinhibins reduce VEGF-induced eNOS phosphorylation through protein phosphatase $2 \mathrm{~A}(\mathrm{PP} 2 \mathrm{~A})$, which is known to dephosphorylate eNOS at Ser1179 (25). As shown in Figure 2, $B$ and C, treatment of BAECs with vasoinhibins increased PP2Alike activity in a dose- and time-dependent manner. The maximal effect was observed after a 1-hour exposure of BAECs to $20 \mathrm{nM}$ vasoinhibins; this time and dose were therefore used in subsequent experiments. We found that the vasoinhibin-stimulated phosphatase activity was prevented by okadaic acid (OA; $50 \mathrm{nM})$, a specific inhibitor of PP2A (26) (Figure 2D). Furthermore, the vasoinhibin effect on PP2A-like activity can be mimicked by rapamycin $(20 \mathrm{nM})$, a known activator of PP2A (27), and OA also inhibited rapamycin-induced $\mathrm{PP} 2 \mathrm{~A}$ activation (Figure $2 \mathrm{D}$ ). These results suggest that vasoinhibins activate PP2A.

We then determined whether inhibition of eNOS phosphorylation by vasoinhibins is mediated by their activation of PP2A. We found that the effect of vasoinhibins on eNOS dephosphorylation was inhibited by OA, thus implicating PP2A (Figure 3B). Using

\section{Figure 3}

Vasoinhibins inhibit VEGF-induced eNOS phosphorylation and eNOS activity via PP2A activation. (A) Line diagrams depicting the different protocols used in experiments represented in B, C, and D. (B) Western blot analysis of eNOS-Ser1179 phosphorylation in BAECs preincubated or not with $50 \mathrm{nM} O A$ for 10 minutes, then with or without $20 \mathrm{nM}$ vasoinhibins for 1 hour, followed or not by a treatment with $5 \mathrm{nM}$ VEGF for 5 minutes as indicated. Total eNOS served as loading control. (C) eNOS activity was determined by the $\left[{ }^{3} \mathrm{H}\right] \mathrm{L}-$-citrulline assay in BAECs incubated or not with $5 \mathrm{nM}$ VEGF, alone or combined with $20 \mathrm{nM}$ vasoinhibins in the presence or absence of $50 \mathrm{nM}$ OA for 1 hour. Values are mean \pm SEM of 5 independent experiments. ${ }^{*} P<0.05$ versus no treatment; ${ }^{* *} P<0.05$ versus VEGF; $\# P<0.05$ versus VEGF and vasoinhibins. (D) Vasopermeability was measured by HRP leakage in BAEC monolayers untreated (ctl) or treated for 48 hours with $50 \mathrm{nM} \mathrm{OA}, 5 \mathrm{nM}$ VEGF, or the combination of VEGF and $20 \mathrm{nM}$ vasoinhibins or VEGF, vasoinhibins, and $\mathrm{OA}$. Values are mean \pm SEM of 3 independent experiments. ${ }^{*} P<0.05$ versus no treatment; ${ }^{* *} P<0.05$ versus VEGF; $\# P<0.05$ versus VEGF and vasoinhibins.

\section{Figure 2}

Vasoinhibins enhance PP2A activity in BAECs. (A) Line diagrams depicting the different protocols used in experiments represented in $\mathbf{B}$, C, and D. PP2A-like activity was measured in BAECs after treatment with increasing doses of vasoinhibins for 1 hour (B) and after incubation with $20 \mathrm{nM}$ vasoinhibins for 1 or 30 minutes or 1,2 , or 3 hours (C). (D) PP2A-like activity was measured in BAECs after 1 hour of incubation in the absence or presence of $20 \mathrm{nM}$ vasoinhibins, $50 \mathrm{nM} O A$, the combination of vasoinhibins and OA, $20 \mathrm{nM}$ rapamycin (Rap), or the combination of rapamycin and OA. Bars correspond to the mean \pm SEM from 4 independent experiments. ${ }^{*} P<0.05$ versus no treatment; ${ }^{*} P<0.05$ versus vasoinhibins or rapamycin alone.

the $\left[{ }^{3} \mathrm{H}\right] \mathrm{L}$-citrulline assay, we showed that vasoinhibins inhibited VEGF-induced eNOS activation and that this effect was blocked by OA. OA alone, on the other hand, had no effect on either basal eNOS activation or VEGF-induced eNOS stimulation (Figure 3C). Next, we asked whether inactivation of PP2A by OA would abrogate the vasoinhibin effect on vasopermeability. We found that treatment of BAECs with OA blocked the ability of vasoinhibin to inhibit VEGF-induced vasopermeability (Figure 3D), and OA alone did not increase basal vasopermeability (Figure 3D). Together, our data showed that vasoinhibins blocked VEGF-induced vasopermeability via PP2A-dependent dephosphorylation and concomitant inactivation of eNOS.

Vasoinhibins inhibit VEGF-induced retinal hemorrbages and vasodilation in vivo. To assess the physiological relevance of our results, we next conducted in vivo studies. Injection of $300 \mathrm{ng}$ VEGF intravitreally (resulting in $260 \mathrm{nM}$ VEGF in the vitreous) in adult male Wistar rats caused multiple hemorrhage areas and vasodilation 24 hours after injection, as shown by staining flat mounts of rat retinas for peroxidase, a marker of red blood cells (Figure 4A, middle panel). Coinjection of $1 \mu \mathrm{g}$ vasoinhibins ( $1 \mu \mathrm{M}$ in the vitreous) with VEGF reduced the vascular changes induced by VEGF (Figure 4A, right

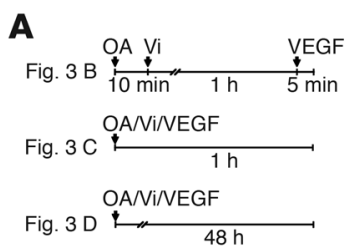

B

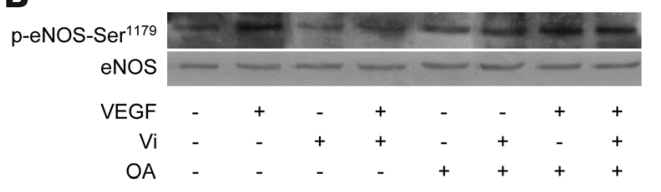

C

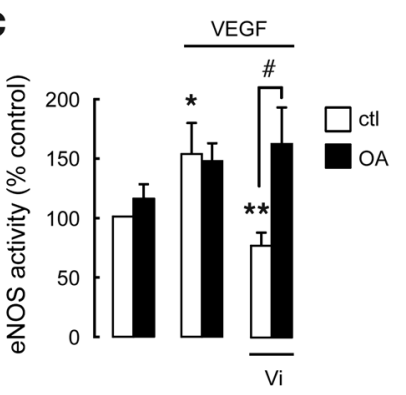

D

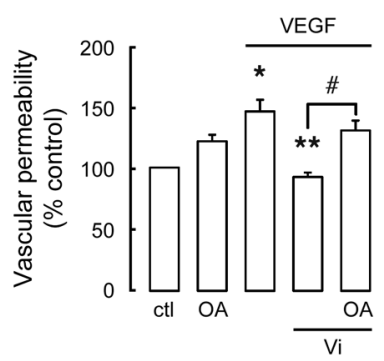


A
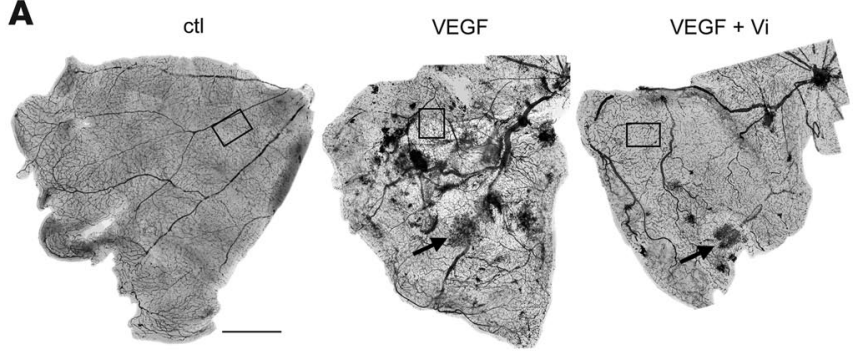

B
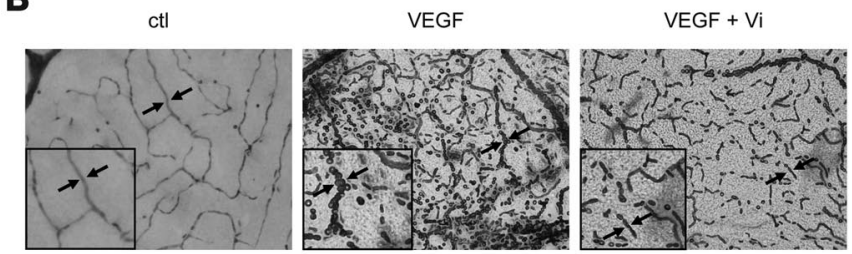

C

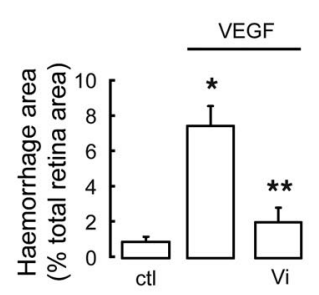

D

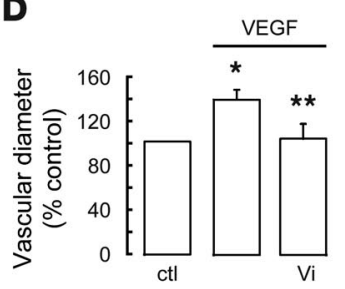

panel). No vascular alterations were observed in retinas from eyes that received a control intravitreal injection of PBS (Figure 4A, left panel). VEGF stimulated vasodilation of major, minor, and capillary vessels. Magnified areas of retinas in Figure 4A illustrate the retinal microcirculation, where VEGF-induced vasodilation allowed erythrocytes to adopt a round morphology (Figure 4B, middle panel versus left panel). Addition of vasoinhibins reduced VEGF-induced dilation of microvessels, thereby preventing the rounding of erythrocytes, which exhibited a shape that was more similar to that of the PBS-treated control (Figure 4B, left versus right panel). Quantification of hemorrhage areas and vessel diameter confirmed that coinjection of vasoinhibins with VEGF reduced both parameters to values similar to those of the controls (Figure 4, C and D). Thus, vasoinhibins inhibit VEGF-induced retinal hemorrhages and vasodilation in vivo.

Dephosphorylation of NOS by vasoinhibins prevents VEGF-induced retinal vasopermeability. Changes in retinal vasopermeability were further investigated using the Evans blue method. VEGF injected intravitreally induced a 4-fold increase in retinal vasopermeability, which was totally blocked by coinjection of vasoinhibins (Figure $5 A)$. Intravitreal injection of vasoinhibins alone, compared with PBS, did not significantly modify retinal vasopermeability (Figure $5 A)$. The contribution of NOS to VEGF-induced retinal vasopermeability was confirmed by the fact that administration of L-NAME $(1.8 \mathrm{mM})$ in the rats' drinking water for 15 days counteracted VEGF action, mimicking the vasoinhibin effect (Figure 5A). Next, OA $(2 \mu \mathrm{M})$ administered intravitreally prevented vasoinhibins from inhibiting VEGF action and caused a stimulation significantly greater than VEGF alone (Figure 5A). Injection of OA alone or combined with VEGF had no significant effect with respect to untreated control or VEGF only, respectively. NOS activity in retinal extracts from rats previously injected intravitreally with the vari-

\section{Figure 4}

Vasoinhibins prevent VEGF-induced retinal hemorrhages and vasodilation in vivo. (A) Representative flat mounts after the peroxidase reaction of a portion of the retina obtained from rats 24 hours after an intravitreous injection of PBS (ctl), $260 \mathrm{nM}$ VEGF, or VEGF and $1 \mu \mathrm{M}$ vasoinhibins. Each retina was digitized with $a \times 20$ objective and tiled to present the entire preparation. Arrows indicate hemorrhage areas. Original magnification, $\times 6$. (B) Magnification of areas indicated by rectangles $(\times 7 ; \times 40$ objective) in the flat mounts shown in $\mathbf{A}$ to illustrate the vasodilation of the microcirculation. Insets in B were magnified by an additional 2-fold. Arrows indicate the width of blood vessels. Quantification of hemorrhage areas (C) and averaged diameter of blood vessels (D) from retina flat mounts from rats injected intravitreally 24 hours earlier with PBS, $260 \mathrm{nM}$ VEGF, or VEGF and $1 \mu \mathrm{M}$ Vi. VEGF and vasoinhibin concentrations refer to the final, intravitreal levels. Values are mean \pm SEM of 6 independent samples. ${ }^{*} P<0.05$ versus control; ${ }^{* \star} P<0.05$ versus VEGF.

ous reagents was then examined by the $\left[{ }^{3} \mathrm{H}\right] \mathrm{L}$-citrulline assay. While injection of vitreous with VEGF activated retinal NOS relative to PBS injection, vasoinhibins abolished the VEGF-induced NOS activity (Figure 5B). To address whether the inhibition of NOS by vasoinhibins could occur in retinal endothelial cells, eNOS activity was determined in RRCECs. VEGF (5 nM) induced a 35\% increase in permeability after 48 hours (Figure 5C), and this increase was blocked by the coadministration of L-NAME $(1 \mathrm{mM})$ or of vasoinhibins in a dose-dependent manner (Figure 5C). While OA (50 nM) did not modify either basal or VEGF-induced eNOS activity, it blocked vasoinhibin inhibition of VEGF-induced eNOS activity in RRCECs (Figure 5D). As shown in Figure 5E, VEGF injection significantly increased retinal eNOS phosphorylation at Ser1179, while vasoinhibins blocked this effect. Quantification normalized to total eNOS content is presented in Figure 5F. Note that vasoinhibins alone did not affect eNOS phosphorylation at Ser1179. These findings established that vasoinhibins protect against VEGFinduced retinal vasopermeability in vivo by activating PP2A, which, in turn, dephosphorylates and inactivates eNOS.

Vasoinhibins block retinal vasopermeability associated with diabetes. We next sought to study the effect of vasoinhibins on retinal vasopermeability associated with diabetic retinopathy. As determined by the Evans blue assay, injection of rats with vitreous from patients with diabetic retinopathy induced an 8 -fold increase in retinal vasopermeability, which was significantly higher than the 2 -fold increase in retinal vasopermeability observed upon injection of nondiabetic vitreous (Figure 6A). These findings are in contrast to those of a previous report (28) showing a 2 -fold increase in vasopermeability in response to vitreous from patients with diabetic retinopathy and no vasopermeability effect of nondiabetic vitreous. In that study, control vitreous was obtained from individuals undergoing vitrectomy, whereas we used vitreous from nondiabetic cadaveric donors. It is possible that postmortem changes in vitreous influence its vasopermeability of properties. Coinjection of vasoinhibins reduced the diabetic vitreous-induced effect on retinal vasopermeability to values comparable to those in eyes injected with the nondiabetic vitreous (Figure 6A). The effect of vasoinhibins on diabetic vitreous-induced retinal vasopermeability was similar to that observed with diabetic retinopathy vitreous in which VEGF was depleted by immunoprecipitation (Figure 6A). VEGF immunoassay showed a 60\% reduction in VEGF in the diabetic and nondiabetic vitreous after immunoprecipitation 
A

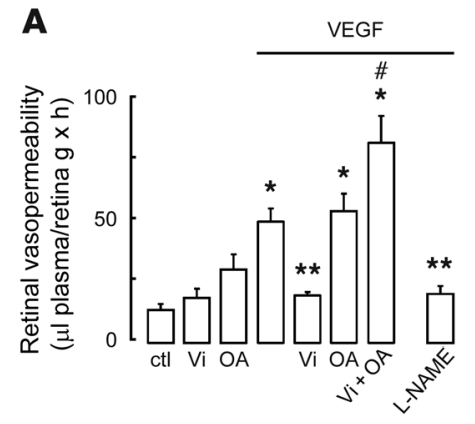

C

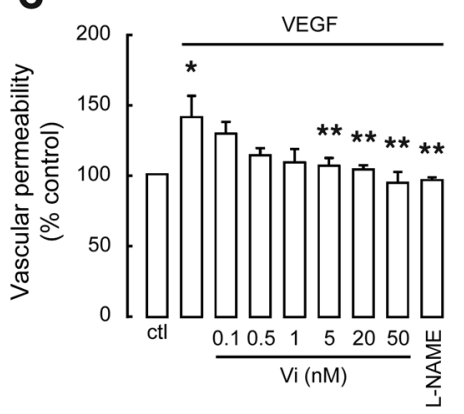

E

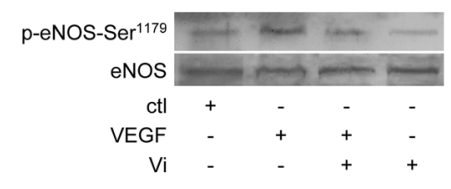

B

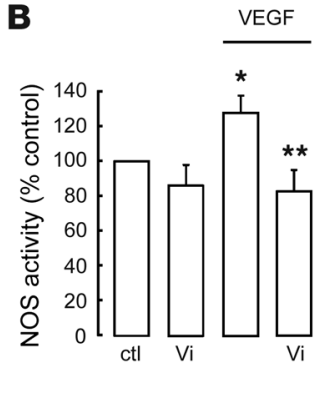

D

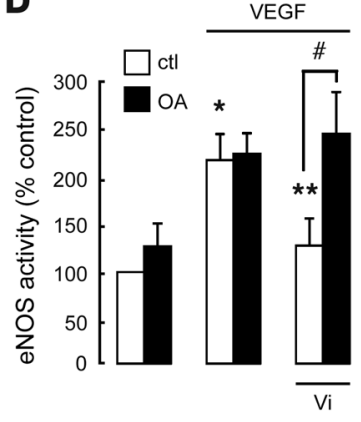

$\mathbf{F}$

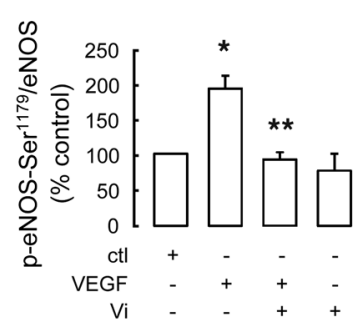

\section{Figure 5}

Vasoinhibins prevent VEGF-induced retinal vasopermeability in vivo via NOS inactivation. (A) Retinal vasopermeability was determined by Evans blue assay of retinal extracts from rats injected intravitreally 24 hours earlier with PBS (ctl), $1 \mu \mathrm{M}$ vasoinhibins; $2 \mu \mathrm{M}$ OA; $260 \mathrm{nM}$ VEGF; VEGF and vasoinhibins; VEGF and OA; or VEGF, vasoinhibins, and OA, as indicated. L-NAME $(1.8 \mathrm{mM})$ was administered in the drinking water for 15 days, and retinas were analyzed for retinal vasopermeability. Values are mean \pm SEM of 10 independent samples. ${ }^{\star} P<0.05$ versus control; ${ }^{*} P<0.05$ versus VEGF; ${ }^{*} P<0.05$ versus vasoinhibins and VEGF. (B) The $\left[{ }^{3} \mathrm{H}\right] \mathrm{L}$-citrulline assay was used to determine NOS activity in retinal extracts from rats injected intravitreally 24 hours earlier with PBS, $1 \mu \mathrm{M}$ vasoinhibins, $260 \mathrm{nM}$ VEGF, or VEGF plus vasoinhibins. Values are mean \pm SEM of 10 independent samples. ${ }^{*} P<0.05$ versus control; ${ }^{* *} P<0.05$ versus VEGF. VEGF and vasoinhibin concentrations refer to the final, intravitreal levels. (C) Vasopermeability was measured by HRP leakage in RRCECs untreated (ctl), treated with either 5 nM VEGF or VEGF combined with $1 \mathrm{mM}$ L-NAME or $0.1,0.5,1,5,20$, or $50 \mathrm{nM}$ vasoinhibins for 48 hours as indicated. Values are mean \pm SEM of 6 independent experiments. ${ }^{\star} P<0.05$ versus untreated control; ${ }^{* *} P<0.05$ versus VEGF. (D) eNOS activity was determined in RRCECs treated with or without 5 nM VEGF alone or together with $20 \mathrm{nM}$ vasoinhibins, in the presence or absence of $50 \mathrm{nM}$ OA for 1 hour. Values are mean \pm SEM of 3 independent experiments. ${ }^{*} P<0.05$ versus no treatment; ${ }^{* \star} P<0.05$ versus VEGF; $\# P<0.05$ versus VEGF and vasoinhibins. (E) Western blot analysis of eNOS-Ser1179 phosphorylation in rat retinas 3 hours after injection with PBS (ctl), VEGF (260 nM), VEGF plus vasoinhibins $(1 \mu \mathrm{M})$, or vasoinhibins alone. Total eNOS served as loading control. (F) Quantification of eNOS phosphorylation by densitometry normalized to total eNOS. Values are mean \pm SEM of 3 independent experiments. ${ }^{*} P<0.05$ versus control; ${ }^{* \star} P<0.05$ versus VEGF.
(Figure 6B). Furthermore, when L-NAME was administered to the rats' drinking water for 15 days, the effect of diabetic vitreous on retinal vasopermeability was reduced to levels equivalent to those after coinjection with vasoinhibins (Figure 6A). Finally, retinal vasopermeability was assessed 2 weeks after diabetes was induced by streptozotocin in rats subjected or not to intravitreous injection of vasoinhibins for 48 hours. As shown in Figure $6 \mathrm{C}$, vasopermeability significantly decreased in retinas of diabetic rats injected with vasoinhibins compared with those injected with PBS. These results suggest that VEGF plays a role in diabetic vitreous-induced retinal vasopermeability and that vasoinhibins block diabetes-associated retinal vasopermeability by interfering with VEGF-induced NO synthesis.

\section{Discussion}

Signs of diabetic retinopathy are evident long before vision loss, and they include retinal microaneurysms, dilated capillary vessels, and hemorrhages. At this stage, the blood vessels leak blood and fluid into the retinal tissue, which results in retinal thickening and formation of hard exudates that lead to reduced vision when the macula is affected $(2,3)$. These changes reflect an imbalance between factors that stimulate and factors that inhibit retinal vasopermeability. Natural inhibitors that play causative roles in diabetic retinal vasopermeability have the potential to be developed for therapeutic application. The present study demonstrates for the first time to our knowledge that vasoinhibins prevent the diabetic retinopathy-associated increase in retinal vasopermeability. This effect involves the inhibition of VEGF-induced eNOS activation due to dephosphorylation of eNOS by PP2A. Our findings suggest that vasoinhibins could be developed as protective agents against diabetic macular edema.

Retinal vasopermeability: VEGF versus vasoinhibins. VEGF is one of the factors that lead to elevated retinal vasopermeability in diabetes. Vascular leakage is associated with upregulation of VEGF in ocular fluids of patients with diabetic retinopathy (29-31). AntiVEGF treatments that were shown to reduce experimental retinal vasopermeability (32) are emerging therapies for the effective treatment of diabetic macular edema $(6,33)$. In this context, the present results unveil what we believe to be a novel action of vasoinhibins as inhibitors of diabetes-associated and VEGF-induced retinal vasopermeability. We demonstrate that intravitreal injection of vasoinhibins prevents excessive retinal vasopermeability in the streptozotocin-induced diabetes rat model, where VEGF plays a major role (34). Also, vasoinhibins block increased retinal vasopermeability induced by the intravitreal injection of VEGF. However, the concentrations of VEGF required by us $(260 \mathrm{nM})$ and by others $(30 \mathrm{nM})(35)$ to increase retinal vasopermeability are much higher than the VEGF levels detected in the vitreous of diabetic patients (220 pM, ref. 29; and 5.8 pM, present study) or of streptozotocininduced diabetic rats (225 pM) (34). Intravitreal delivery may limit 

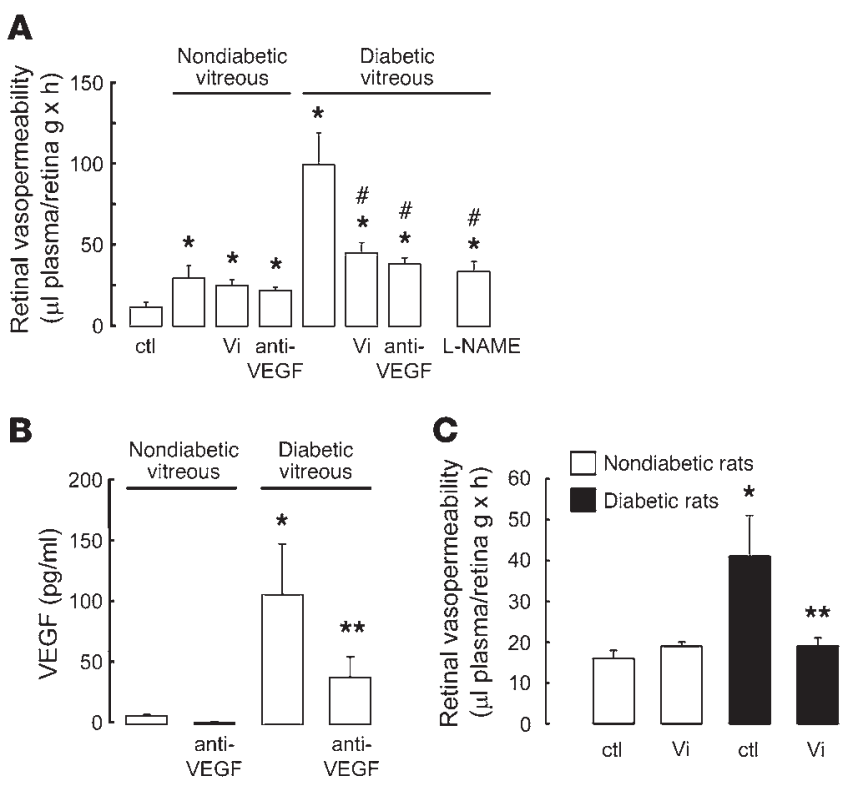

Figure 6

Vasoinhibins prevent retinal vasopermeability induced by human diabetic vitreous. (A) Evans blue evaluation of retinal vasopermeability in retinal extracts from rats injected intravitreally with PBS (ctl, $n=5$ ), nondiabetic vitreous $(2 \mu \mathrm{l}, n=5)$, nondiabetic vitreous plus vasoinhibins $(1 \mu \mathrm{M}, n=5)$, nondiabetic vitreous depleted of VEGF (anti-VEGF, $2 \mu \mathrm{l}, n=3)$, diabetic vitreous $(2 \mu \mathrm{l}, n=6)$, diabetic vitreous plus vasoinhibins ( $1 \mu \mathrm{M}, n=6$ ), or diabetic vitreous depleted of VEGF (anti-VEGF, $2 \mu l, n=3)$. The same VEGF-depleted vitreous was used for the experiments in A and B. L-NAME (1.8 mM) was administered in drinking water for 15 days, and rat retinas that had been intravitreally injected with diabetic vitreous were subsequently analyzed for retinal vasopermeability. Values are mean \pm SEM of $n$ independent experiments. ${ }^{*} P<0.05$ versus ctl; ${ }^{*} P<0.05$ versus diabetic vitreous. (B) Analysis of VEGF concentration ( $\mathrm{pg} / \mathrm{ml}$ ) by ELISA in nondiabetic vitreous, nondiabetic vitreous depleted of VEGF (anti-VEGF), diabetic vitreous, and diabetic vitreous depleted of VEGF (anti-VEGF). The VEGF content of the vitreous samples was reduced by immunoprecipitation with antiVEGF antibody. Values are mean \pm SEM of 3 independent vitreous samples. ${ }^{\star} P<0.05$ versus nondiabetic vitreous; ${ }^{\star \star} P<0.05$ versus diabetic vitreous. (C) Evans blue evaluation of retinal vasopermeability in retinal extracts from nondiabetic rats and diabetic rats (induced by streptozotocin), injected with PBS or vasoinhibins $(1 \mu \mathrm{M})$. Values are mean \pm SEM of 6 independent retinal extracts. ${ }^{*} P<0.05$ versus PBSinjected nondiabetic rats; ${ }^{*} P<0.05$ versus $P B S$-injected diabetic rats. Vasoinhibin concentrations refer to the final, intravitreal values.

the accessibility and tissue distribution of VEGF, which could account for such a discrepancy. Also, the composition of the vitreous proteome changes with diabetes (28), and molecular interactions with other vasopermeability factors or cofactors could help potentiate the effect of the endogenous VEGF so that it is active at much lower concentrations. In any event, we also show that vasoinhibins counteract the effect of the vitreous from patients with diabetic retinopathy, resulting in vasopermeability levels similar to those observed with diabetic vitreous in which VEGF was depleted by immunoprecipitation. These data indicate that vasoinhibins protect against the increased retinal vasopermeability associated with diabetes by opposing the effects of endogenous VEGF. Consistent with this interpretation, we found that the small but significant effect of the vitreous from nondiabetic individuals on retinal vasopermeability was independent of VEGF and was not affected by vasoinhibins. This may imply the presence of other factors, e.g., carbonic anhydrase (28), that also contribute to basal retinal vasopermeability. Because control vitreous was obtained from cadaveric donors, upregulation of these factors may be due to possible postmortem changes, although such changes did not appear to modify VEGF levels. Finally, we report that vasoinhibins counteract VEGF-induced dilation of retinal blood vessels, a known effect of VEGF (36) that is among the earliest manifestations of diabetic retinopathy (37).

We believe the demonstration that vasoinhibins block the vasopermeability and vasodilation effects of VEGF to be novel and potentially important. Vasoinhibins were previously shown to inhibit growth factor- (VEGF and bFGF) and IL-1 $\beta$-induced endothelial cell migration $(38,39)$ and proliferation $(40)$ and to promote endothelial cell apoptosis (41). Current studies extend vasoinhibin function to inhibition of increases in vascular permeability that accompany almost all states of angiogenesis (42), including those in areas of diseased tissue in angiogenesis-related disorders such as diabetic retinopathy. The role of vasoinhibins as physio(patho)logical regulators of retinal vasculature is supported by their presence in the normal rat retina (22) and in the subretinal fluid and fibrovascular membranes of patients with retinopathy of prematurity (20). Blockage of vasoinhibins by the intravitreal injection of small, interfering RNA or neutralizing antibodies stimulates vessel growth and dilation in the retina (22) and inhibits apoptosis-mediated regression of intraocular blood vessels during development (20). Notably, gene transfer of vasoinhibins via an adenoviral vector reduces angiogenesis in murine ischemia-induced retinopathy (43). These observations indicate that vasoinhibins are endogenous antiangiogenic, vasoconstrictive, and antivasopermeability factors in retina. Thus, vasoinhibins can be used to ameliorate multiple features of diabetic retinopathy. In line with this, PRL levels increase in the circulation and in ocular fluids of patients with advanced retinopathy of prematurity, and this increase was proposed to result in higher levels of intraocular vasoinhibins that can counteract the progression of the disease (20).

Mechanism of vasoinhibin action. Vasoinhibin actions described here are consistent with their effect on inhibiting VEGF-induced NO synthesis (21), which is one of the mechanisms by which VEGF induces angiogenesis, vasodilation, and vasopermeability (16-18). We show that L-NAME, a NOS inhibitor, mimicked the inhibitory effect of vasoinhibins on VEGF-induced vasopermeability in vivo and in vitro. Moreover, L-NAME prevented elevated retinal vasopermeability in response to the vitreous from patients with diabetic retinopathy, and it reduced retinal vasopermeability to a level similar to that following VEGF depletion or vasoinhibin treatment. Vasoinhibins indeed impaired VEGF-induced NOS activity in the retina and, because vasoinhibins also inhibit eNOS activity in RRCECs, this action can occur directly on retinal blood vessels. At present there are 2 mechanisms by which vasoinhibins might inhibit eNOS activation. We previously proposed that vasoinhibins, by interfering with the mobilization of intracellular calcium, could prevent the calcium-dependent activation of eNOS (21). Here, we show that vasoinhibins block eNOS activity by inducing its dephosphorylation at Ser1179 via PP2A stimulation. VEGF is known to stimulate eNOS activity via PI3K/Aktdependent phosphorylation of eNOS at Ser1179 (bovine eNOS) or Ser1177 (human eNOS) (44). Phosphorylation of eNOS is an important mechanism that promotes eNOS-vascular functions, 
Table 1

Characteristics of the subjects studied

\begin{tabular}{|c|c|c|c|c|c|c|c|}
\hline Group & Subject & $\begin{array}{l}\text { Age } \\
(\mathrm{yr})\end{array}$ & $\begin{array}{c}\text { Type of } \\
\text { diabetes }\end{array}$ & $\begin{array}{c}\text { Duration of } \\
\text { diabetes (yr) }\end{array}$ & $\begin{array}{c}\text { HbA1c } \\
(\%)^{A}\end{array}$ & $\begin{array}{c}\text { Mean blood } \\
\text { pressure }(\mathrm{mmHg})\end{array}$ & Indications \\
\hline \multicolumn{8}{|c|}{ Males with severe ${ }^{\mathrm{B}}$ proliferative diabetic retinopathy } \\
\hline & 1 & 71 & 2 & 27 & 8 & 100 & Vitreous hemorrhages \\
\hline & 2 & 61 & 2 & 15 & 7 & 100 & Retinal detachment \\
\hline & 3 & 65 & 2 & 15 & 7 & 86.6 & Vitreous hemorrhages and macular edema \\
\hline & 4 & 28 & 1 & 18 & 10.9 & 146.7 & Vitreous hemorrhages \\
\hline & 5 & 47 & 2 & 10 & 9 & 100 & Retinal detachment \\
\hline & 6 & 47 & 2 & 5 & 8 & 100 & Vitreous hemorrhages and macular edema \\
\hline
\end{tabular}

Male nondiabetic cadaveric donors $\mathrm{C}$

\begin{tabular}{|c|c|c|c|c|c|c|}
\hline 1 & 32 & NA & NA & NA & NA & $N A$ \\
\hline 2 & 21 & NA & NA & NA & NA & NA \\
\hline 3 & 58 & NA & $N A$ & NA & NA & NA \\
\hline 4 & 33 & NA & NA & NA & NA & NA \\
\hline 5 & 76 & NA & NA & NA & NA & NA \\
\hline
\end{tabular}

A HbA1c, hemoglobin A1c blood test result. ${ }^{B}$ Ref. 68 . C Donors died by accidental death, and samples were obtained $12.5 \pm 0.5$ hours postmortem. NA, not applicable.

i.e., vasodilation, vasopermeability, and angiogenesis (15-17), and it may represent a switch for their control under physiological and pathological conditions. Transgenic mice expressing only a phosphomimetic form of eNOS (due to point mutation of Ser1179 to Asp1179) respond better to vasorelaxing agents and display increased blood reperfusion during ischemia, reducing the severity of strokes (45). Also, shear stress and several vasoactive factors and hormones such as insulin (46) and estrogens (47) induced eNOS Ser1179 phosphorylation. Dephosphorylation of this residue may represent a common mechanism by which vasoinhibins could counteract the effects of several vasoactive substances.

Our work also demonstrates the prominent role of PP2A in the antipermeability effect of vasoinhibins in vivo and in vitro. The observation that OA, in the presence of vasoinhibins, permits VEGFinduced vasopermeability in the retina and in cultured endothelial cells implicates an OA-sensitive phosphatase (26). OA had no significant effect under unstimulated conditions or when combined with VEGF. This lack of effect implies that PP2A is not activated under basal conditions or in response to VEGF. The stimulatory effect of $\mathrm{OA}$ in the presence of VEGF and vasoinhibins suggests that activation of PP2A by vasoinhibins can reduce vasopermeability through additional pathways besides eNOS inactivation, such as dephosphorylation of occludin (48). By reducing eNOS phosphorylation via PP2A activation, endostatin was shown to impair endothelial cell migration and angiogenesis but not vasopermeability (49). Similar to angiostatin (50), endostatin can prevent increased vasopermeability by reducing paracellular permeability at the level of occludin expression (51). Vasoinhibins act directly on endothelial cells through a still-unidentified, saturable, high-affinity binding site distinct from the PRL receptor (52), and the mechanism by which vasoinhibins activate PP2A is unknown. Actually, the mechanisms by which several stimulators regulate PP2A activity are unclear $(53,54)$.

The negative regulation of the proliferative effects of VEGF by vasoinhibins may not involve PP2A, since PP2A and vasoinhibins block activation of Ras upstream and downstream of the Shc/ Grb2/Sos adapter protein complex, respectively $(55,56)$. On the other hand, activation of PP2A-induced eNOS dephosphorylation by vasoinhibins may help counteract the VEGF activation of the MAPK pathway. NO reverses the inhibition of VEGF-induced endothelial cell proliferation by vasoinhibins (21) and promotes the activation of the MAPK pathway (16). Also, vasoinhibins inhibit the p38 MAPK/STAT1/IRF-1 pathway, thereby attenuating inducible NOS/NO production in endothelial cells (57), and PP2A inhibits the p38 MAPK pathway by dephosphorylating the upstream activator of p38, MKK3 (58). With regard to the effects of PP2A on endothelial cell apoptosis, PP2A interferes with survival pathways by acting as a major Akt and Bcl-2 phosphatase (54, 59), but it remains to be determined whether vasoinhibins modify the phosphorylation of Akt and/or Bcl-2.

Clinical implications. Until recently, the main treatment for diabetic retinopathy including diabetic macular edema has been laser photocoagulation, which protects from further vision loss but seldom restores visual acuity (4). As the excess of VEGF in the retina plays a major role in the increased retinal vasopermeability, an early complication of diabetes (37) and a major cause of diabetic macular edema (2), several new anti-VEGF therapies, either targeted directly against VEGF or toward its downstream effects, have recently emerged (6). Despite an encouraging clinical benefit (33), treatment must still be optimized for routine use, and combination therapy is currently regarded as effective for improvement of visual acuity (60). Thus, identification and characterization of new molecules able to counteract VEGF action are necessary. Our findings strongly support the relevance of eNOS dephosphorylation for the prevention of retinal vasopermeability associated with diabetic retinopathy and identify a prominent association between eNOS-related VEGF signaling and vasoinhibins. Promoting vasoinhibin activity to counteract VEGF and reduce excessive retinal vasopermeability in diabetic retinopathy emerges from this study as a potentially powerful therapeutic approach for effective treatment of diabetic macular edema and the various vasoproliferative retinopathies.

\section{Methods}

Reagents. Rat vasoinhibin (corresponding to a $16-\mathrm{kDa}$ fragment of PRL) used in in vivo experiments was generated by enzymatic proteolysis of rat PRL with a particulate fraction from rat mammary glands, followed by gel filtration and carbamidomethylation as previously described (61). Recom- 
binant human vasoinhibins (corresponding to a $14-\mathrm{kDa}$ fragment of PRL) used in cell culture experiments were generated by site-directed mutagenesis as previously described (62). A vasoinhibin concentration of $1 \mu \mathrm{M}$ was used in vivo because it fully blocked increased retinal vasopermeability elicited by VEGF and diabetic vitreous, as determined by a dose-response curve (data not shown). Human vitreous specimens were obtained after surgery on 6 subjects with diabetic retinopathy. The specimens were obtained with the approval of the Institutional Review Board of the Hospital "Dr. Luís Sánchez Bulnes," and all subjects were treated in accordance with the tenets of the Declaration of Helsinki. All patients provided written informed consent before collection of samples. Vitreous samples from nondiabetic cadaveric donors were used as controls $(n=5)$. The Medical Forensic Service (SEMEFO) of Mexico approved the collection and use of samples. All vitreous samples were tested individually. Table 1 summarizes the characteristics of all subjects studied. Recombinant human VEGF $\left(\mathrm{rhVEGF}_{165}\right)$ was a gift from Genentech. The lowest doses of VEGF having a significant effect in vitro and in vivo were $5 \mathrm{nM}$ and $260 \mathrm{nM}$, respectively. OA (Millipore) was used at $50 \mathrm{nM}$ according to the manufacturer's instructions; rapamycin and L-NAME were from Sigma-Aldrich. Doses of rapamycin and L-NAME in in vitro experiments were selected as previously reported (27).

Cell culture. BAECs were grown in DMEM supplemented with $1 \%$ penicillin/ streptomycin, 1\% Fungizone, 5\% FBS (Gibco; Invitrogen), 5\% fetal calf serum (Equitech-Bio Inc.), and $10 \mathrm{mM}$ thymidine (Sigma-Aldrich). RRCECs were isolated from rat retinas and cultured as previously described (63). Prior to each treatment, cells were cultured in DMEM with 0.5\% FBS for 18 hours.

Animals. Male Wistar rats were maintained and treated in accordance with the rules and regulations of the Association for Research in Vision and Ophthalmology Statement for the Use of Animals in Ophthalmic and Vision Research. All animal experiments were approved by the Bioethics Committee of the Institute of Neurobiology, Universidad Nacional Autónoma de México. All experimental procedures used aseptic techniques. Male Wistar rats of 200-250 g were anesthetized with $70 \%$ ketamine and $30 \%$ xylazine $(1 \mu \mathrm{l} / \mathrm{g}$ body weight intraperitoneally) for intravitreous injection. After 24 hours, animals were sacrificed, and blood samples and retinas were collected. Diabetes was induced with streptozotocin $(60 \mathrm{mg} / \mathrm{kg}$ ) for 2 weeks as reported previously (64). Animals with glucose levels greater than $250 \mathrm{mg} / \mathrm{dl}$ were used.

Intravitreal injections. Intravitreal injection in rat eyes was performed as previously described (22). One eye was injected with VEGF (300 ng per eye, i.e., $260 \mathrm{nM}$, as the estimated volume of rat vitreous is $60 \mu \mathrm{l}$; ref. 65) and the contralateral eye with the combination of VEGF $(260 \mathrm{nM})$ and vasoinhibins $(1 \mu \mathrm{g}$ per eye, i.e., $1 \mu \mathrm{M})$. For the control groups, the animals were injected in one eye with PBS and in the contralateral eye with vasoinhibins $(1 \mu \mathrm{M})$. For the experiments with human samples, diabetic or nondiabetic vitreous was injected into the eye $(2 \mu \mathrm{l})$. The contralateral eye received the same human fluid together with vasoinhibins $(1 \mu \mathrm{g})$. In diabetic rats, PBS or vasoinhibin $(1 \mu \mathrm{g})$ injections were performed after 2 weeks of hyperglycemia as reported previously (64). The final volume of injection was adjusted to $4 \mu \mathrm{l}$.

Capillary network staining. The capillary network was delineated by staining the enclosed red blood cells using a peroxidase reaction, as previously described (40).

Permeability assay in vitro. The vascular permeability assay was performed as previously described (66) with some modifications. Polycarbonate filters (8.0- $\mu \mathrm{m}$ pore size and 24 -well plate) (Corning Inc.) were used. HRP type VI-A (MW, 44,000; Sigma-Aldrich) was used as a standard and as the marker to measure monolayer permeability. $\operatorname{HRP}(0.5 \mu \mathrm{g} / \mathrm{ml})$ was added to the upper filter compartment. After a 48-hour incubation of BAEC or RRCEC monolayers on the filters at $37^{\circ} \mathrm{C}, 30 \mu \mathrm{l}$ of the culture medium collected from the lower compartment was added to $860 \mu \mathrm{l}$ of a reaction buffer containing $50 \mathrm{mM}$ monobasic sodium phosphate and $5 \mathrm{mM}$ guaiacol (Sigma-Aldrich). The reaction was started by adding $100 \mu \mathrm{l}$ hydrogen peroxide $(25 \mathrm{mM})$ and was incubated for 30 minutes at room temperature. The absorbance at $450 \mathrm{~nm}$ was determined.

Measurement of hemorrhages and blood vessel dilation in vivo. The visualization of the retinal vascular network and vasodilation assessment were performed as previously described (22).

Measurement of capillary permeability in vivo. One day after intravitreous injection, the Evans blue dye technique was used as previously described (67).

ELISA for VEGF. The levels of VEGF in human vitreous samples were assessed according to the manufacturer's instructions (Biosource; Invitrogen). The assay has a sensitivity of $5 \mathrm{pg} / \mathrm{ml}$. VEGF was immunoprecipitated by incubating $100 \mu \mathrm{l}$ of human vitreous with $1 \mu \mathrm{l}$ of VEGF antibody (catalog AF564; R\&D Systems) overnight and then with $10 \mu \mathrm{l}$ protein $\mathrm{G}$ agarose (Invitrogen) for 1 hour. Samples were then centrifuged at 16,000 $\mathrm{g}$ for 10 minutes at $4^{\circ} \mathrm{C}$, and the supernatant was collected for the ELISA.

Western blot analysis. BAECs were pretreated with OA $(50 \mathrm{nM})$ for $10 \mathrm{~min}$ utes, vasoinhibins ( $20 \mathrm{nM}$ ) for 1 hour, or VEGF $(5 \mathrm{nM})$ for 1,5 , or $10 \mathrm{~min}$ utes, as indicated in the legends. Next, cell samples were processed as previously described (63). Polyclonal anti-phospho-Ser1179-eNOS antibody (1:1,000 dilution; catalog 9571; Cell Signaling Technology) was incubated overnight, and the antigen-antibody complex was detected using HRPcoupled secondary antibodies (catalog 111-035-003; Jackson ImmunoResearch Laboratories Inc.) and enhanced chemiluminescence (ECL; SuperSignal West Pico Chemiluminescent Substrate; Pierce Biotechnology). Membranes were then stripped and probed with monoclonal anti-eNOS (1:1,000 dilution; catalog 33-4500, Zymed Laboratories Inc.; Invitrogen) and alkaline phosphatase second antibody kit (Bio-Rad).

L-Citrulline assay. eNOS activity was measured in BAECs and in RRCECs as previously reported (21). For tissue assays, rats were euthanized 24 hours after intravitreal injections, and retinas were dissected and homogenized in extraction buffer (320 mM sucrose, 20 mM HEPES, 1 mM EDTA, 1 mM DTT, 10 $\mu \mathrm{l} / \mathrm{ml}$ leupeptin, and $2 \mu \mathrm{l} / \mathrm{ml}$ aprotinin, $\mathrm{pH}$ 7.2). Protein concentration was evaluated using the Bradford assay (Bio-Rad). The reaction was carried out in reaction buffer $\left(50 \mathrm{mM}\right.$ HEPES, $1 \mathrm{mM}$ NADPH, $1.25 \mathrm{mM} \mathrm{CaCl}_{2}, 1 \mu \mathrm{M}$ FAD, $1 \mu \mathrm{M} \mathrm{FMN}$, and $10 \mu \mathrm{g} / \mathrm{ml}$ calmodulin) and initiated by the addition of $1 \mu \mathrm{Ci} /$ $\mathrm{ml}\left[{ }^{3} \mathrm{H}\right] \mathrm{L}$-arginine to $50 \mu \mathrm{g}$ of total retinal extract protein in a final volume of $100 \mu$ l. After 25 minutes, ice-cold stop medium was added (50 mM HEPES, pH 5.5, and 4 mM EDTA). Retinal extracts in the extraction buffer described above were applied onto 1-ml columns of Dowex AG50WX8. [ $\left.{ }^{3} \mathrm{H}\right] \mathrm{L}$-citrulline was eluted with $1 \mathrm{ml}$ water and quantified by liquid scintillation counting.

Phosphatase assay. The Serine/Threonine Phosphatase Assay was performed according to manufacturer's instructions (Promega). After different treatments indicated in the legends, cells were lysed in a PP2A-specific buffer $(25 \mathrm{mM}$ Tris- $\mathrm{HCl}$ at $\mathrm{pH} 7.5,10 \mathrm{mM} \beta$-mercaptoethanol, 2 mM EDTA, $0.1 \mathrm{mM}$ PMSF, $1 \mu \mathrm{M}$ pepstatin A, and $10 \mu \mathrm{g} / \mathrm{ml}$ aprotinin). Endogenous phosphate was removed using spin columns, and the lysates were incubated with the serine/threonine phosphopeptide in PP2A buffer at $30^{\circ} \mathrm{C}$ for 15 minutes. PP2A-like activity, reported as free phosphate released, was calculated from the absorbance at $595 \mathrm{~nm}$ of a molybdate-malachite green-phosphate complex formed after addition of $1 \mathrm{ml}$ molybdate dye solution and $10 \mu \mathrm{l}$ of molybdate dye additive (Promega).

Statistics. Values are expressed as mean \pm SEM. Statistical significance between groups was determined by ANOVA followed by the unpaired 2 -tailed Student's $t$ test. The statistical analyses were performed using SigmaStat 7.0 software (SYSTAT). Differences in means with $P<0.05$ were considered statistically significant.

\section{Acknowledgments}

We thank Fernando López-Barrera, Gabriel Nava, Daniel Mondragón, Antonio Prado, and Martín García for their technical assistance and Dorothy D. Pless for critically editing the manu- 
script. This study was supported by Consejo Nacional de Ciencia y Tecnología (grants 49292 and SALUD-2004-CO2-16) and Universidad Nacional Autónoma de México grant IN202406.

Received for publication November 15, 2007, and accepted in revised form April 9, 2008.

1. Williams, R., et al. 2004. Epidemiology of diabetic retinopathy and macular oedema: a systematic review. Eye. 18:963-983.

2. Knudsen, S.T., et al. 2002. Macular edema reflects generalized vascular hyperpermeability in type 2 diabetic patients with retinopathy. Diabetes Care. 25:2328-2334.

3. Hammes, H.P. 2005. Pericytes and the pathogenesis of diabetic retinopathy. Horm. Metab. Res. 37(Suppl. 1):39-43.

4. Bloomgarden, Z.T. 2007. Screening for and managing diabetic retinopathy: current approaches. Am.J. Health Syst. Pharm. 64:S8-S14.

5. Caldwell, R.B., et al. 2003. Vascular endothelial growth factor and diabetic retinopathy: pathophysiological mechanisms and treatment perspectives. Diabetes Metab. Res. Rev. 19:442-455.

6. Emerson, M.V., and Lauer, A.K. 2007. Emerging therapies for the treatment of neovascular agerelated macular degeneration and diabetic macular edema. BioDrugs. 21:245-257.

7. Miyamoto, K., et al. 2000. Vascular endothelial growth factor (VEGF)-induced retinal vascular permeability is mediated by intercellular adhesion molecule-1 (ICAM-1). Am. J. Pathol. 156:1733-1739.

8. Tolentino, M.J., et al. 1996. Intravitreous injections of vascular endothelial growth factor produce retinal ischemia and microangiopathy in an adult primate. Ophthalmology. 103:1820-1828.

9. Aiello, L.P., et al. 1997. Vascular endothelial growth factor-induced retinal permeability is mediated by protein kinase $\mathrm{C}$ in vivo and suppressed by an orally effective beta-isoform-selective inhibitor. Diabetes. 46:1473-1480.

10. Clermont, A.C., Aiello, L.P., Mori, F., Aiello, L.M., and Bursell, S.E. 1997. Vascular endothelial growth factor and severity of nonproliferative diabetic retinopathy mediate retinal hemodynamics in vivo: a potential role for vascular endothelial growth factor in the progression of nonproliferative diabetic retinopathy. Am. J. Ophthalmol. 124:433-446.

11. Boulton, M., Foreman, D., Williams, G., and McLeod, D. 1998. VEGF localisation in diabetic retinopathy. Br. J. Ophthalmol. 82:561-568.

12. Kevil, C.G., Payne, D.K., Mire, E., and Alexander, J.S. 1998. Vascular permeability factor/vascular endothelial cell growth factor-mediated permeability occurs through disorganization of endothelial junctional proteins. J. Biol. Chem. 273:15099-15103.

13. Antonetti, D.A., et al. 1998. Vascular permeability in experimental diabetes is associated with reduced endothelial occludin content: vascular endothelial growth factor decreases occludin in retinal endothelial cells. Penn State Retina Research Group. Diabetes. 47:1953-1959.

14. Feng, Y., et al. 1999. VEGF-induced permeability increase is mediated by caveolae. Invest. Ophthalmol. Vis. Sci. 40:157-167.

15. Walford, G., and Loscalzo, J. 2003. Nitric oxide in vascular biology. J. Thromb. Haemost. 1:2112-2118.

16. Ziche, M., and Morbidelli, L. 2000. Nitric oxide and angiogenesis. J. Neurooncol. 50:139-148.

17. Fukumura, D., et al. 2001. Predominant role of endothelial nitric oxide synthase in vascular endothelial growth factor-induced angiogenesis and vascular permeability. Proc. Natl. Acad. Sci. U. S. A. 98:2604-2609.
Address correspondence to: Carmen Clapp, Instituto de Neurobiología, Universidad Nacional Autónoma de México, Campus UNAM-Juriquilla, 76230 Querétaro, Mexico. Phone: (52-442) 2381028; Fax: (52-442) 238-1005; E-mail: clapp@servidor.unam.mx.

Celina García and Jorge Aranda contributed equally to this work.
18. Tilton, R.G., et al. 1999. Role for nitric oxide in the hyperpermeability and hemodynamic changes induced by intravenous VEGF. Invest. Ophthalmol. Vis. Sci. 40:689-696.

19. Clapp, C., Aranda, J., Gonzalez, C., Jeziorski, M.C., and Martinez de la Escalera, G. 2006. Vasoinhibins: endogenous regulators of angiogenesis and vascular function. Trends Endocrinol. Metab. 17:301-307.

20. Duenas, Z., et al. 2004. Prolactin in eyes of patients with retinopathy of prematurity: implications for vascular regression. Invest. Ophthalmol. Vis. Sci. 45:2049-2055.

21. Gonzalez, C., et al. 2004. 16K-prolactin inhibits activation of endothelial nitric oxide synthase, intracellular calcium mobilization, and endothelium-dependent vasorelaxation. Endocrinology. 145:5714-5722.

22. Aranda, J., et al. 2005. Prolactins are natural inhibitors of angiogenesis in the retina. Invest. Ophthalmol. Vis. Sci. 46:2947-2953.

23. Rees, D.D., Palmer, R.M., Hodson, H.F., and Moncada, S. 1989. A specific inhibitor of nitric oxide formation from $\mathrm{L}$-arginine attenuates endothelium-dependent relaxation. Br. J. Pharmacol. 96:418-424.

24. Boo, Y.C., and Jo, H. 2003. Flow-dependent regulation of endothelial nitric oxide synthase: role of protein kinases. Am. J. Physiol. Cell Physiol. 285:C499-C508.

25. Michell, B.J., et al. 2001. Coordinated control of endothelial nitric-oxide synthase phosphorylation by protein kinase $\mathrm{C}$ and the cAMP-dependent protein kinase. J. Biol. Chem. 276:17625-17628.

26. Boudreau, R.T., and Hoskin, D.W. 2005. The use of okadaic acid to elucidate the intracellular role(s) of protein phosphatase 2A: lessons from the mast cell model system. Int. Immunopharmacol. 5:1507-1518.

27. Hartley, D., and Cooper, G.M. 2002. Role of mTOR in the degradation of IRS-1: regulation of PP2A activity. J. Cell. Biochem. 85:304-314.

28. Gao, B.B., et al. 2007. Extracellular carbonic anhydrase mediates hemorrhagic retinal and cerebral vascular permeability through prekallikrein activation. Nat. Med. 13:181-188.

29. Aiello, L.P., et al. 1994. Vascular endothelial growth factor in ocular fluid of patients with diabetic retinopathy and other retinal disorders. N. Engl.J. Med. 331:1480-1487.

30. Simo, R., et al. 2002. Free insulin growth factor-I and vascular endothelial growth factor in the vitreous fluid of patients with proliferative diabetic retinopathy. Am. J. Ophthalmol. 134:376-382.

31. Funatsu, H., et al. 2006. Vitreous levels of pigment epithelium-derived factor and vascular endothelial growth factor are related to diabetic macular edema. Ophthalmology. 113:294-301.

32. Qaum, T., et al. 2001. VEGF-initiated blood-retinal barrier breakdown in early diabetes. Invest. Ophthalmol. Vis. Sci. 42:2408-2413.

33. Arevalo, J.F., et al. 2007. Primary intravitreal bevacizumab (Avastin) for diabetic macular edema: results from the Pan-American Collaborative Retina Study Group at 6-month follow-up. Ophthalmology. 114:743-750.

34. Amrite, A.C., Ayalasomayajula, S.P., Cheruvu, N.P., and Kompella, U.B. 2006. Single periocular injection of celecoxib-PLGA microparticles inhibits diabetes-induced elevations in retinal PGE2,
VEGF, and vascular leakage. Invest. Ophthalmol. Vis. Sci. 47:1149-1160.

35. Ojima, T., et al. 2006. EphrinA1 inhibits vascular endothelial growth factor-induced intracellular signaling and suppresses retinal neovascularization and blood-retinal barrier breakdown. Am.J. Pathol. 168:331-339.

36. Ashrafpour, H., et al. 2004. Vasodilator effect and mechanism of action of vascular endothelial growth factor in skin vasculature. Am. J. Physiol. Heart Circ. Physiol. 286:H946-H954.

37. Kristinsson, J.K., Gottfredsdottir, M.S., and Stefansson, E. 1997. Retinal vessel dilatation and elongation precedes diabetic macular oedema. Br. J. Ophthalmol. 81:274-278.

38. Lee, H., Struman, I., Clapp, C., Martial, J., and Weiner, R.I. 1998. Inhibition of urokinase activity by the antiangiogenic factor $16 \mathrm{~K}$ prolactin: activation of plasminogen activator inhibitor 1 expression. Endocrinology. 139:3696-3703.

39. Lee, S.H., Kunz, J., Lin, S.H., and Yu-Lee, L.Y. 2007. $16-\mathrm{kDa}$ prolactin inhibits endothelial cell migration by down-regulating the Ras-Tiam1-Rac1-Pak1 signaling pathway. Cancer Res. 67:11045-11053.

40. Clapp, C., Martial, J.A., Guzman, R.C., RentierDelure, F., and Weiner, R.I. 1993. The 16-kilodalton $\mathrm{N}$-terminal fragment of human prolactin is a potent inhibitor of angiogenesis. Endocrinology. 133:1292-1299.

41. Martini, J.F., et al. 2000. The antiangiogenic factor $16 \mathrm{~K}$ PRL induces programmed cell death in endothelial cells by caspase activation. Mol. Endocrinol. 14:1536-1549.

42. Bates, D.O., Hillman, N.J., Williams, B., Neal, C.R., and Pocock, T.M. 2002. Regulation of microvascular permeability by vascular endothelial growth factors. J. Anat. 200:581-597.

43. Pan, H., et al. 2004. Molecular targeting of antiangiogenic factor 16K hPRL inhibits oxygen-induced retinopathy in mice. Invest. Ophthalmol. Vis. Sci. 45:2413-2419.

44. Six, I., Kureishi, Y., Luo, Z., and Walsh, K. 2002. Akt signaling mediates VEGF/VPF vascular permeability in vivo. FEBS Lett. 532:67-69.

45. Atochin, D.N., et al. 2007. The phosphorylation state of eNOS modulates vascular reactivity and outcome of cerebral ischemia in vivo. J. Clin. Invest. 117:1961-1967.

46. Montagnani, M., Chen, H., Barr, V.A., and Quon, M.J. 2001. Insulin-stimulated activation of eNOS is independent of Ca2+ but requires phosphorylation by Akt at $\operatorname{Ser}(1179)$. J. Biol. Chem. 276:30392-30398.

47. Hisamoto, K., et al. 2001. Estrogen induces the Aktdependent activation of endothelial nitric-oxide synthase in vascular endothelial cells. J. Biol. Chem. 276:3459-3467.

48. Seth, A., Sheth, P., Elias, B.C., and Rao, R. 2007. Protein phosphatases $2 \mathrm{~A}$ and 1 interact with occludin and negatively regulate the assembly of tight junctions in the CACO-2 cell monolayer. J. Biol. Chem. 282:11487-11498.

49. Urbich, C., et al. 2002. Dephosphorylation of endothelial nitric oxide synthase contributes to the anti-angiogenic effects of endostatin. FASEB J. 16:706-708.

50. Shyong, M.P., et al. 2007. Reduction of experimental diabetic vascular leakage by delivery of angiostatin with a recombinant adeno-associated virus 
vector. Mol. Vis. 13:133-141.

51. Campbell, M., et al. 2006. Involvement of MAPKs in endostatin-mediated regulation of blood-retinal barrier function. Curr. Eye Res. 31:1033-1045.

52. Clapp, C., and Weiner, R.I. 1992. A specific, high affinity, saturable binding site for the 16-kilodalton fragment of prolactin on capillary endothelial cells. Endocrinology. 130:1380-1386.

53. Junttila, M.R., Li, S.P., and Westermarck, J. 2007. Phosphatase-mediated crosstalk between MAPK signaling pathways in the regulation of cell survival. FASEB J. 22:954-965.

54. Ruvolo, P.P. 2003. Intracellular signal transduction pathways activated by ceramide and its metabolites. Pharmacol. Res. 47:383-392.

55. D'Angelo, G., et al. 1999. 16K human prolactin inhibits vascular endothelial growth factor-induced activation of Ras in capillary endothelial cells. Mol. Endocrinol. 13:692-704.

56. Ugi, S., Imamura, T., Ricketts, W., and Olefsky, J.M 2002. Protein phosphatase $2 \mathrm{~A}$ forms a molecular complex with Shc and regulates Shc tyrosine phosphorylation and downstream mitogenic signaling. Mol. Cell. Biol. 22:2375-2387.

57. Lee, S.H., et al. 2005. 16-kDa prolactin down-regu- lates inducible nitric oxide synthase expression through inhibition of the signal transducer and activator of transcription 1/IFN regulatory factor-1 pathway. Cancer Res. 65:7984-7992.

58. Prickett, T.D., and Brautigan, D.L. 2007. Cytokine activation of p38 mitogen-activated protein kinase and apoptosis is opposed by alpha- 4 targeting of protein phosphatase $2 \mathrm{~A}$ for site-specific dephosphorylation of MEK3. Mol. Cell. Biol. 27:4217-4227.

59. Millward, T.A., Zolnierowicz, S., and Hemmings, B.A. 1999. Regulation of protein kinase cascades by protein phosphatase 2A. Trends Biochem. Sci. 24:186-191.

60. Mohamed, Q., Gillies, M.C., and Wong, T.Y. 2007. Management of diabetic retinopathy: a systematic review. JAMA. 298:902-916.

61. Clapp, C. 1987. Analysis of the proteolytic cleavage of prolactin by the mammary gland and liver of the rat: characterization of the cleaved and $16 \mathrm{~K}$ forms. Endocrinology. 121:2055-2064.

62. Galfione, M., et al. 2003. Expression and purification of the angiogenesis inhibitor $16-\mathrm{kDa}$ prolactin fragment from insect cells. Protein Expr. Purif. 28:252-258.
63. Ochoa, A., et al. 2001. Expression of prolactin gene and secretion of prolactin by rat retinal capillary endothelial cells. Invest. Ophthalmol. Vis. Sci. 42:1639-1645.

64. Navaratna, D., McGuire, P.G., Menicucci, G., and Das, A. 2007. Proteolytic degradation of VE-cadherin alters the blood-retinal barrier in diabetes. Diabetes. 56:2380-2387.

65. Guo, L., et al. 2007. Targeting amyloid-beta in glaucoma treatment. Proc. Natl. Acad. Sci. U. S. A. 104:13444-13449.

66. Wang, Y., Gu, Y., Granger, D.N., Roberts, J.M., and Alexander, J.S. 2002. Endothelial junctional protein redistribution and increased monolayer permeability in human umbilical vein endothelial cells isolated during preeclampsia. Am.J. Obstet. Gynecol. 186:214-220.

67. Xu, Q., Qaum, T., and Adamis, A.P. 2001. Sensitive blood-retinal barrier breakdown quantitation using Evans blue. Invest. Ophthalmol. Vis. Sci. 42:789-794.

68. Wilkinson, C.P., et al. 2003. Proposed international clinical diabetic retinopathy and diabetic macular edema disease severity scales. Ophthalmology. 110:1677-1682. 